\title{
An Efficient Technique for Solving Inhomogeneous Electromagnetic Inverse Scattering Problems
}

\author{
Mohamed Elkattan*
}

\begin{abstract}
The electromagnetic inverse scattering approach seeks to obtain the electric characteristics of a scatterer using information about the source and the scattered data. The inverse scattering problem usually suffers from limited knowledge about the scatterer used, which makes its solution more challenging than the forward problem. This paper presents an inversion approach to estimating the unknown electric properties of a two- and three-dimensional inhomogeneous scatterer. The presented approach considers the inverse scattering problem as a global minimization problem with a meshless forward formulation for the computation of the scattered electromagnetic field. Various simulated annealing cooling schedules are applied and assessed to solve the problem, and the results of several case studies are presented for both two- and three-dimensional electromagnetic inverse scattering problems.
\end{abstract}

Key Words: Electromagnetic, Forward Problem, Inhomogeneous Scatterer, Inversion, Meshless.

\section{INTRODUCTION}

Electromagnetic surveying systems are widely used for environmental and engineering studies $[1,2]$. In the electromagnetic method, a source is used to produce an incident wave, where interaction between the wave and the investigated object leads to generation of a scattered wave. Data are gathered at the receiver to obtain information to predict the physical properties of the investigated configuration through solving the inverse scattering problem [3].

Inverse scattering problems have several applications in research areas such as archeology, geophysical prospecting, and medical imaging $[4,5]$. These types of problems suffer from a limited amount of information about the variables to be estimated [6, 7]. However, solutions to such problems must be accurate to get a reasonable estimate, and a reasonable estimate is mainly affected by the efficiency of the forward problem formulation. Several techniques are available for forward problem formulation, such as the finite-difference and finite-element methods $[8,9]$ and the boundary element method [10]. However, such conventional numerical methods have limitations, including numerical polarization and dispersion [11, 12], inaccurate representation of decaying fields, and integrals with singularity or hyper-singularity $[13,14]$. Thus, one of the objectives of this paper is to provide an accurate forward modeling method that overcomes some of these limitations and difficulties.

Another objective of this paper is to develop an inversion methodology that benefits from the optimal setting of the forward formulation to solve both two- and three-dimensional (2D and 3D) electromagnetic inverse scattering problems in inhomogeneous media. In our approach, the inverse problem is treated as a sampling problem using the simulated annealing

Manuscript received April 19, 2019 ; Revised August 6, 2019 ; Accepted December 2, 2019. (ID No. 20190419-022J)

Department of Electronics, Nuclear Materials Authority, Cairo, Egypt.

"Corresponding Author: Mohamed Elkattan (e-mail: emtiazegf@hotmail.com)

This is an Open-Access article distributed under the terms of the Creative Commons Attribution Non-Commercial License (http://creativecommons.org/licenses/by-nc/4.0) which permits unrestricted non-commercial use, distribution, and reproduction in any medium, provided the original work is properly cited.

(c) Copyright The Korean Institute of Electromagnetic Engineering and Science. All Rights Reserved. 
method without affecting the nonlinear nature of the problem. Moreover, several simulated annealing algorithms were designed and compared with different case studies to ensure the approach's efficiency in terms of estimation error.

\section{FORWARD FORMULATION}

An efficient forward algorithm for calculating scattered fields from input model parameters (i.e., permittivity and conductivity) is required to solve an inversion problem [8]. Most inversion approaches have a link between the forward and inverse problems in order to evaluate the fitness of the predicted parameters for the proposed model [9].

Forward problem techniques are always based on a previously selected configuration, and the effectiveness of the forward configuration strongly influences the efficiency of the inversion approach. Approaches used for modeling electromagnetic forward problems fall into two main categories: mesh-based and meshless approaches [15].

Most commercial simulators use mesh-based approaches to solve forward problems. Mesh-based approaches have several limitations because they must truncate the domain of computation, which introduces unphysical nonreflecting boundaries [16, 17]. They also create a large grid with too many cells to get an accurate solution, especially if the structure is lossy and contain evanescent fields [18]. These limitations mean that these approaches suffer from long simulation times [19].

Meshless approaches can be used for the forward formulation of Maxwell's equations through the expansion of fields using Fourier transform or Gauss-Hermite functions [20]. However, these approaches involve many integrals that have to be evaluated numerically, so they are computationally expensive. In this paper, a meshless approach will be adopted that avoids such integrals; therefore, reducing the computational complexity of the solution, and that locates accurate estimates of the model with a reasonable error margin.

\section{Two-Dimensional Problem}

In this paper, we will consider the 2D model shown in Fig. 1, where an object of permittivity $\epsilon_{r 2}$ and conductivity $\sigma_{2}$ is

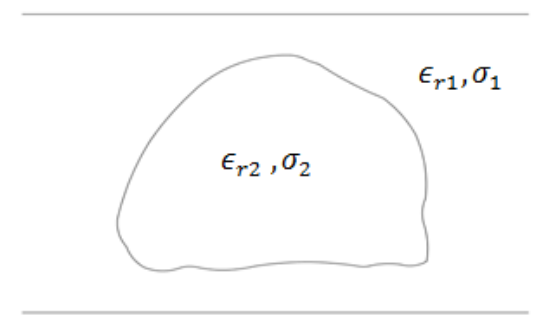

Fig. 1. General model of the 2D electromagnetic inverse scattering problem considered in this paper. buried inside a slab with permittivity $\epsilon_{r 1}$ and conductivity $\sigma_{1}$. For the $2 \mathrm{D}$ problem, the electric polarization current can be represented as [21]:

$$
\begin{gathered}
J(x, z)=Q(x, z)\left[E_{i}(x, z)+\int_{\infty}^{-\infty} \int_{0}^{L} G\left(x, z, x^{\prime}, z^{\prime}\right) J\left(x^{\prime}, z^{\prime}\right) d z^{\prime} d x^{\prime}\right], \\
\infty>x, x^{\prime}>-\infty, L \geq z, z^{\prime} \geq 0
\end{gathered}
$$

where $E_{i}(x, z)$ is the incident electric field, $(x, z)$ is the Cartesian coordinate of an observation point, $\left(x^{\prime}, z^{\prime}\right)$ is the Cartesian coordinate of the polarization current, and $L$ is the slab thickness. $G=\frac{i}{4} H_{0}^{(1)}\left(x, z, x^{\prime}, z^{\prime}\right)$, where $H_{0}^{(1)}($.$) is the$ Hankel function of the first type and zeroth order. $Q(x, z)$ can be expanded to be written as [22]:

$$
Q(x, z)=i w \mu Q^{\prime}(x, z),
$$

where

$$
Q^{\prime}(x, z)=-i w\left(\epsilon(x, z)-\epsilon_{0}\right)+\sigma(x, z)
$$

For the 2D case of one-layer slab with an object inside it, we can write $Q(x, z)$ as:

$$
Q(x, z)=Q(\text { slab })-[Q(\text { slab })-Q(\text { object })] \Omega(s)
$$

where $\Omega(s)$ is the indicator to classify the object from the slab. $\Omega(s)=0$ indicates the slab while $\Omega(s)=1$ indicates the object, and

$$
\begin{aligned}
Q(\text { slab }) & =k_{\text {slab }}^{2}-k_{0}^{2} \\
Q(\text { object }) & =k_{\text {object }}^{2}-k_{0}^{2}
\end{aligned}
$$

where $k_{0}=w \sqrt{\mu_{0} \epsilon_{0}}$ is the background medium. Hence,

$$
\begin{gathered}
k_{\text {slab }}^{2}(x, z)=w^{2} \mu_{0} \epsilon_{0} \epsilon_{r}(\text { slab })+i w \mu_{0} \sigma(\text { slab }) \\
k_{\text {object }}^{2}(x, z)=w^{2} \mu_{0} \epsilon_{0} \epsilon_{r}(\text { object })+i w \mu_{0} \sigma(\text { object }) .
\end{gathered}
$$

One approach to solve this problem is to use Fourier transform and $\left\{\psi_{n}\right\}$ orthogonality properties [23], but by this representation, overlap integrals in $k_{x}$ and $\mathrm{n}$ are required. These integrals have a very high computational cost. To avoid this cost, we propose another approach that avoids all overlap integrals: the point-by-point approach. In this approach, we get the scatterer's polarization of current expansion coefficients $a_{n}\left(k_{x}\right)$ in the form of:

$$
\begin{aligned}
& \sum_{n} \int d k_{x} \exp \left(i k_{x} x\right) a_{n}\left(k_{x}\right) \psi_{n}\left(z, k_{x}\right) \\
& =Q(x, z) E_{i}(x, z) \\
& +Q(x, z) \sum_{n} \int d k_{x} \frac{i}{2 k_{z}} \exp \left(i k_{x} x\right) a_{n}\left(k_{x}\right) \lambda_{n}\left(k_{x}\right) \psi_{n}\left(z, k_{x}\right)
\end{aligned}
$$

where $k_{z}=\sqrt{k_{0}^{2}-k_{x}^{2}}$ stands for the $2 \mathrm{D}$ case, $\lambda_{n}\left(k_{x}\right)$ st- 
ands for eigenvalue, and $\psi_{n}\left(z, k_{x}\right)$ represents a complete set. Here, the estimated current expansion coefficients are $a_{n}\left(k_{x}\right)$ at $a([n k], 1)$ values, that is, $N_{\text {modes }} \times K_{\text {spatial harmonics }}$. We therefore need to identify $O \times P$ points of $z$ and $x$ directions, which can be selected within the scatterer. Once defined, the current expansion coefficient equation can be rewritten as:

$$
\begin{gathered}
\sum_{n} \sum_{k_{x}} \exp \left(i k_{x} x_{P}\right) a_{n}\left(k_{x}\right) \psi_{n}\left(z_{O}, k_{x}\right) \\
=\frac{1}{\delta k_{x}} Q\left(x_{P}, z_{O}\right) E_{i}\left(x_{P}, z_{O}\right) \\
+Q\left(x_{P}, z_{O}\right) \sum_{n} \sum_{k_{x}} \frac{i}{2 k_{z}} \exp \left(i k_{x} x_{P}\right) a_{n}\left(k_{x}\right) \lambda_{n}\left(k_{x}\right) \psi_{n}\left(z_{o}, k_{x}\right) \\
\forall o, p
\end{gathered}
$$

Here, integrals are approximated to $\Sigma$ with a step $\delta k_{x}$. Now we can define a linear system to get the estimated current expansion coefficients to be:

$$
\begin{aligned}
& M_{1}([o p],[n k]) a([n k], 1) \\
& \quad=c([o p], 1)+M_{2}([o p],[n k]) a([n k], 1)
\end{aligned}
$$

where

$$
M_{1}([o p],[n k])=\exp \left(i k_{x} x_{P}\right) \psi_{n}\left(z_{O}, k_{x}\right),
$$

and

$$
\begin{aligned}
& M_{2}([o p],[n k])= \\
& Q\left(x_{P}, z_{O}\right) \frac{i}{2 k_{z}} \exp \left(i k_{x} x_{P}\right) \lambda_{n}\left(k_{x}\right) \psi_{n}\left(z_{O}, k_{x}\right),
\end{aligned}
$$

and

$$
c([o p], 1)=\frac{1}{\delta k_{x}} Q\left(x_{P}, z_{O}\right) E_{i}\left(x_{P}, z_{O}\right) .
$$

Current expansion coefficients a can then be computed as:

$$
\left[M_{1}-M_{2}\right] a=c .
$$

Once we compute the coefficients $\left\{a_{n}\left(k_{x}\right)\right\}$, the scattered electric field's distribution is obtained at the receiver for a given $k_{x}$ as:

$$
E_{z r}\left(k_{x}, 0\right)=i \omega \mu_{0} \quad \sum_{n} \frac{i}{2 k_{z}} a_{n}\left(k_{x}\right) \lambda_{n}\left(k_{x}\right) \psi\left(0, k_{x}\right) .
$$

The $2 \mathrm{D}$ problem was modeled by frequency domain finite element analysis using the COMSOL Multiphysics software package. The incident field was a linearly polarized plane wave normalized by $\frac{i}{2 k_{z}}$ at one megahertz under a scattering boundary condition for the structure shown in Fig. 1, with $\epsilon_{r 1}=8, \epsilon_{r 2}=4$ and $\sigma_{1}=0.002, \sigma_{2}=0.005$. The calculated (using Eq. (16)) and the simulated (using COMSOL) scattered electric field results were compared at 11 wavenumbers $\left(k_{x}=0,0.11 k_{0}, 0.22 k_{0}, \ldots, 1.1 k_{0}\right)$, and the results are presented in Fig. 2. The diamonds and circles in the figure rep-

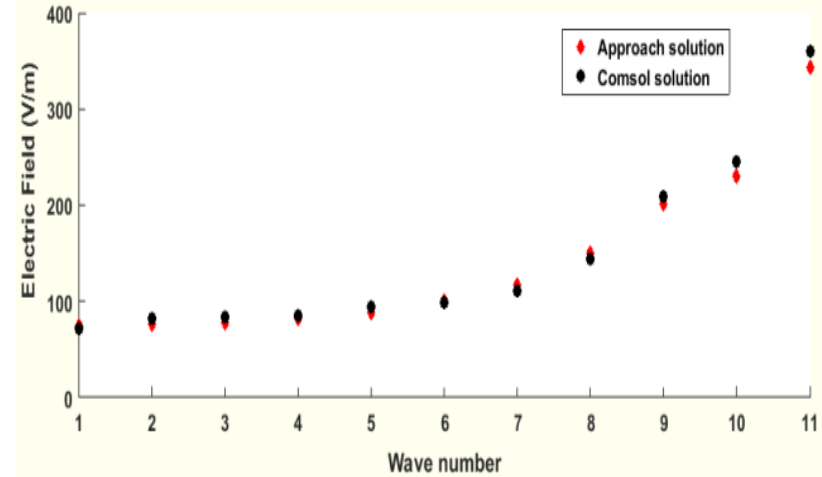

Fig. 2. Calculated (using Eq. (16)) and simulated (using COMSOL) scattered electric field results at 11 wavenumbers for the $2 \mathrm{D}$ problem.

resent the field computations of the proposed method and the finite element simulation. As shown in Fig. 2, the solution of the proposed approach is very close to the finite element analysis solution.

\section{Three-Dimensional Problem}

In order to extend the proposed solution to the $3 \mathrm{D}$ case, the model used in this paper is presented in Fig. 3, where a sphere of permittivity $\epsilon_{r 2}$ and conductivity $\sigma_{2}$ is buried inside a box with permittivity $\epsilon_{r 1}$ and conductivity $\sigma_{1}$. Here, the scatterer polarization current expansion coefficients $a_{n}\left(k_{x}, k_{y}\right)$ can be written in the form of [24]:

$$
\begin{aligned}
& \sum_{n} \iint d k_{x} d k_{y} \exp \left(i k_{x} x\right) \exp \left(i k_{y} y\right) a_{n}\left(k_{x}, k_{y}\right) \psi_{n}\left(z, k_{x}, k_{y}\right) \\
& =Q(x, y, z) E_{i}(x, y, z) \\
& +Q(x, y, z) \sum_{n} \iint d k_{x} d k_{y} \frac{i}{2 k_{z}} \exp \left(i k_{x} x\right) \exp \left(i k_{y} y\right) \\
& a_{n}\left(k_{x}, k_{y}\right) \lambda_{n}\left(k_{x}, k_{y}\right) \psi_{n}\left(z, k_{x}, k_{y}\right)
\end{aligned}
$$

where $k_{z}=\sqrt{k_{0}^{2}-k_{x}^{2}-k_{y}^{2}}$ stands for the $3 \mathrm{D}$ case, $\lambda_{n}$ $\left(k_{x}, k_{y}\right)$ stands for eigenvalue, and $\psi_{n}\left(z, k_{x}, k_{y}\right)$ represents a complete set. Here, the estimated current expansion coefficients are $a_{n}\left(k_{x}, k_{y}\right)$ at $a\left(\left[n k_{x} k_{y}\right], 1\right)$ value $N_{\text {modes }} \times$ $K x_{\text {spatial harmonics }} \times K y_{\text {spatial harmonics. }}$. Here, we need to

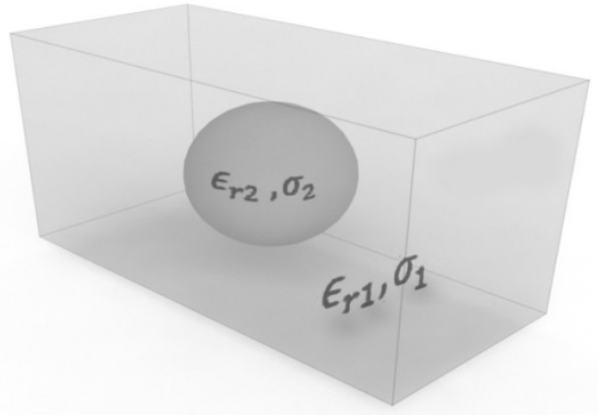

Fig. 3. General model of the 3D electromagnetic inverse scattering problem considered in this paper. 
identify $O \times F \times P$ points of $z, y$, and $x$ directions, which can be selected within the scatterer all together. Once defined, the current expansion coefficient equation can be rewritten as:

$$
\begin{aligned}
& \sum_{n} \sum_{k_{x}} \sum_{k_{y}} \exp \left(i k_{x} x_{P}\right) \exp \left(i k_{y} y_{f}\right) a_{n}\left(k_{x}, k_{y}\right) \psi_{n}\left(z_{O}, k_{x}, k_{y}\right) \\
& =\frac{1}{\delta k_{x} \delta k_{y}} Q\left(x_{P}, y_{f}, z_{O}\right) E_{i}\left(x_{P}, y_{f}, z_{O}\right) \\
& +Q\left(x_{P}, y_{f}, z_{O}\right) \sum_{n} \sum_{k_{x}} \sum_{k_{y}} \frac{i}{2 k_{z}} \exp \left(i k_{x} x_{P}\right) \exp \left(i k_{y} y_{f}\right) a_{n}\left(k_{x}, k_{y}\right) \\
& \lambda_{n}\left(k_{x}, k_{y}\right) \psi_{n}\left(z_{O}, k_{x}, k_{y}\right) \quad \forall o, p, f
\end{aligned}
$$

where integrals are approximated to $\Sigma$ with steps $\delta k_{x}$ and $\delta k_{y}$. By this representation, all overlap integrals in $k_{x}, k_{y}$, and $n$ are avoided. This reduces the computational cost dramatically.

Now we can define a linear system to get the estimated current expansion coefficients to be:

$$
\begin{gathered}
M_{1}\left([o p f],\left[n k_{x} k_{y}\right]\right) a\left(\left[n k_{x} k_{y}\right], 1\right) \\
=c([o p f], 1)+M_{2}\left([o p f],\left[n k_{x} k_{y}\right]\right) a\left(\left[n k_{x} k_{y}\right], 1\right)
\end{gathered}
$$

where

$$
\begin{gathered}
M_{1}\left([o p f],\left[n k_{x} k_{y}\right]\right) \\
=\exp \left(i k_{x} x_{P}\right) \exp \left(i k_{y} y_{f}\right) \psi_{n}\left(z_{O}, k_{x}, k_{y}\right) \\
M_{2}\left([o p f],\left[n k_{x} k_{y}\right]\right) \\
=Q\left(x_{P}, y_{f}, z_{O}\right) \frac{i}{2 k_{z}} \exp \left(i k_{x} x_{P}\right) \exp \left(i k_{y} y_{f}\right) \\
\lambda_{n}\left(k_{x}, k_{y}\right) \psi_{n}\left(z_{O}, k_{x}, k_{y}\right) \\
c([o p f], 1)=\frac{1}{\delta k_{x} \delta k_{y}} Q\left(x_{P}, y_{f}, z_{O}\right) E_{i}\left(x_{P}, y_{f}, z_{O}\right)
\end{gathered}
$$

Then the current expansion coefficient, $a$, can be computed as:

$$
\left[M_{1}-M_{2}\right] a=c .
$$

Once we compute the coefficients $\left\{a_{n}\left(k_{x}, k_{y}\right)\right\}$, the scattered electric field's distribution is obtained at the receiver for a given $k_{x}$ and $k_{y}$ as:

$$
E_{z r}\left(k_{x}, k_{y}, 0\right)=i \omega \mu_{0} \sum_{n} \frac{i}{2 k_{z}} a_{n}\left(k_{x}, k_{y}\right) \lambda_{n}\left(k_{x}, k_{y}\right) \psi\left(0, k_{x}, k_{y}\right)
$$

The 3D problem was modeled by frequency domain finite element analysis using the COMSOL Multiphysics software with the same conditions and structure shown in Fig. 3. The calculated (using Eq. (24)) and the simulated (using COMSOL) scattered electric field results were compared at 11 wavenumbers $\left(k_{x}=0,0.11 k_{0}, \ldots ., 1.1 k_{0}\right.$ and $\left.k_{y}=0,0.11 k_{0}, \ldots 1.1 k_{0}\right)$.

The results are presented in Fig. 4; the diamonds and circles

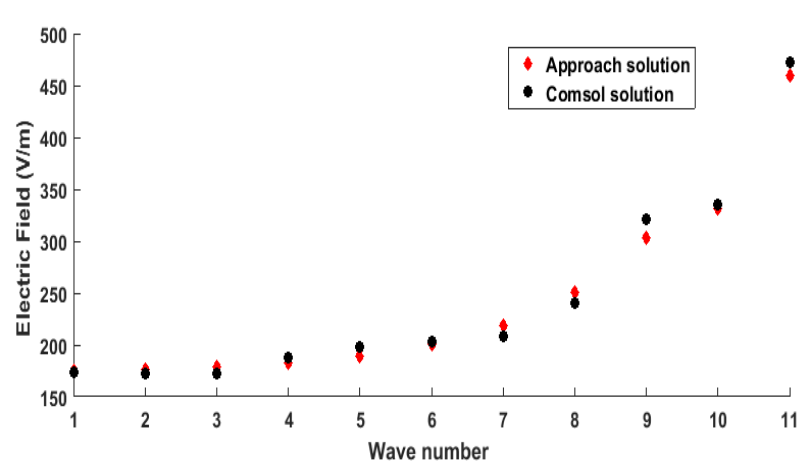

Fig. 4. Calculated (using Eq. (24)) and simulated (using COMSOL) scattered electric field results at 11 wavenumbers for the $2 \mathrm{D}$ problem.

represent the field computations of the proposed method and the finite element simulation. As shown in Fig. 4, the solution of the proposed approach is very close to the finite element analysis solution.

\section{INVERSION APPROACH}

The inversion technique aims to estimate the physical variables that tolerably fit the scattered data [25]. In electromagnetic problems, responses from the model in the form of a scattered electric field are nonlinear functions of the proper model's physical variables [26]. The physical variables of the model used can be predicted using nonlinearized or linearized inversion algorithms [27]. In this paper, the inversion approach uses the developed forward solver repetitively as an integral part of the inverse technique.

The proposed approach rearranges the nonlinear inversion process to find a solution of a set of linear equations. However, the final inversion equation is still nonlinear. This is done by modeling the electromagnetic inverse problem as a global optimization problem, where the multivariable function of the problem is iteratively minimized using the simulated annealing technique $[28,29]$.

Using simulated annealing in the inversion methodology allows for a straightforward insertion of the a priori information into the forward model. Furthermore, the proposed inversion approach does not require the starting solutions to represent good estimates of the exact configuration of the structure. In this paper, optimization of the unknown electrical variables is conducted through an objective function, $O b$, in the form of:

$$
O b=\sum_{k=1}^{K}\left|E_{m}^{n}-E_{c}^{n}\right|^{2}
$$

where $E_{m}^{n}$ is the measured electrical field collected at the $k$ th wavenumber, for a total of $K$ wavenumbers, and $E_{c}^{n}$ is the electric field computed at those wavenumbers based on what the $2 M$ material properties might be (the permittivities and conduc- 
tivities in our case). The objective function is therefore $2 M-$ dimensional:

$$
\left.O b=\mathcal{F}\left(\left\{\varepsilon_{r m}\right\},\left\{\sigma_{m}\right\}\right), m=1: M\right)
$$

where $\varepsilon_{r m}$ and $\sigma_{m}$ are the relative dielectric constant and conductivity of the $m^{t h}$ object. The simulated annealing algorithm starts with initial estimates for the object's electrical properties, driven by a priori knowledge of the possible range of the model's physical parameters; this is called the current estimates.

The algorithm then generates new estimates with a random distance from the current estimates in the search space. This distance is selected by a probability distribution with a scale depending on the current temperature. Afterwards, the algorithm determines whether the new estimates are better or worse than the current estimates by evaluating the objective function, $O b$, for both the current and the new estimates.

If the objective function value of the new estimates is less than that of the current estimates, the new estimates are accepted in place of the current estimates. However, to avoid being trapped in the local minima, the algorithm can still accept new estimates that raise the value of the objective function in place of the current estimates. Thus, the new estimate of the object's electrical properties is accepted either if it is better than the current estimate or if it is worse than the current estimates but with a certain probability value. This probability is controlled by two main factors: the temperature and the difference between the current value and the new value of the objective function, and it can be defined by an acceptance function:

$$
F=\frac{1}{1+\exp \left(\frac{O b_{\text {new }}-O b_{\text {current }}}{T}\right)},
$$

where $O b_{\text {new }}$ is the objective function value of the new estimates, $O b_{\text {current }}$ is the objective function value of the current estimates, and $T$ is the current temperature. By accepting worse estimates based on an acceptance function, the algorithm will be able to explore globally for more possible solutions. However, during the algorithm iterations to find the best estimate, the simulated annealing algorithm systematically decreases the probability of accepting worse estimates by applying the designed cooling schedule $T_{c}$, wherein the temperature decreases, leading to a lesser acceptance probability. In this paper, three simulated annealing cooling schedules are designed, implemented, and compared for performance. The first is exponential, where the cooling schedule is given by [30]:

$$
T_{c}=a^{c} T_{0}
$$

where $c=1,2,3 \ldots$ is the iteration number, $T_{0}$ is the initial temperature, and $a$ is the cooling rate. The second is called the fast cooling schedule and is given by:

$$
T_{c}=\frac{T_{0}}{c+1}
$$

The third is called the Boltzmann cooling schedule, and is given by:

$$
T_{c}=\frac{T_{0}}{\ln (c+1)} .
$$

Here we use $T_{0}=100^{\circ} \mathrm{C}$ and $a=0.95$ for the three cooling schedules. In this paper, the different simulated annealing techniques were used to minimize the error between the predicted and computed electrical variables, aiming to reach an optimum error of $0 \%$. However, if the algorithm cannot reach that goal, stopping criteria of 10,000 iterations is implied. After the stopping criterion is achieved, the algorithm outputs the results that realize the value nearest to the optimum.

\section{RESULTS AND DISCUSSION}

In this paper, for both the $2 \mathrm{D}$ and $3 \mathrm{D}$ problems, we selected 10 possible structures from which to construct 10 case studies. Table 1 summarizes the possible permittivity and conductivity ranges of these structures. To measure the efficiency of the proposed inversion technique, we identify a wideness factor that

\begin{tabular}{|c|c|c|c|c|}
\hline \multicolumn{2}{|c|}{ Case study no. } & \multirow{2}{*}{$\begin{array}{c}\begin{array}{c}\text { Structure } \\
\text { no. }\end{array} \\
1\end{array}$} & \multirow{2}{*}{$\begin{array}{c}\begin{array}{c}\text { Relative } \\
\text { permittivity, } \varepsilon_{r}\end{array} \\
5\end{array}$} & \multirow{2}{*}{$\begin{array}{c}\text { Conductivity, } \sigma \\
0.0008\end{array}$} \\
\hline 1 & Slab/box & & & \\
\hline & Object & 2 & 10 & 0.0013 \\
\hline \multirow[t]{2}{*}{2} & Slab/box & 4 & 12 & 0.04 \\
\hline & Object & 3 & 7 & 0.008 \\
\hline \multirow[t]{2}{*}{3} & Slab/box & 3 & 6 & 0.004 \\
\hline & Object & 5 & 2.10 & 0.0025 \\
\hline \multirow[t]{2}{*}{4} & Slab/box & 3 & 10 & 0.0033 \\
\hline & Object & 1 & 7 & 0.0000125 \\
\hline \multirow[t]{2}{*}{5} & Slab/box & 3 & 11 & 0.001 \\
\hline & Object & 6 & 20 & 0.02 \\
\hline \multirow[t]{2}{*}{6} & Slab/box & 6 & 25 & 0.1 \\
\hline & Object & 8 & 5 & 0.0005 \\
\hline \multirow[t]{2}{*}{7} & Slab/box & 7 & 60 & $2.2 \times 10^{-4}$ \\
\hline & Object & 6 & 40 & 0.0166 \\
\hline \multirow[t]{2}{*}{8} & Slab/box & 7 & 90 & $4 \times 10^{-4}$ \\
\hline & Object & 9 & 25 & $1.6667 \times 10^{-4}$ \\
\hline \multirow[t]{2}{*}{9} & Slab/box & 7 & 80 & $3.33 \times 10^{-4}$ \\
\hline & Object & 8 & 4 & $1.11 \times 10^{-4}$ \\
\hline \multirow[t]{2}{*}{10} & Slab/box & 10 & 30 & 0.01 \\
\hline & Object & 2 & 15 & 0.002 \\
\hline
\end{tabular}
quantifies the wideness of each physical parameter range around the average. The wideness factor represents a priori knowledge

Table 1. Physical properties of the 10 structures used in this paper along with their wideness factors 
Table 2. Physical properties of the ten forward case studies used in this paper

\begin{tabular}{ccccc}
\hline $\begin{array}{c}\text { Struc- } \\
\text { ture } \\
\text { no. }\end{array}$ & $\begin{array}{c}\text { Relative } \\
\text { permittivity, } \\
\varepsilon_{r}\end{array}$ & $\begin{array}{c}\varepsilon_{r} \text { wide- } \\
\text { ness fac- } \\
\text { tor }(\%)\end{array}$ & Conductivity, $\sigma$ & $\begin{array}{c}\sigma \text { wide- } \\
\text { ness fac- } \\
\text { tor }(\%)\end{array}$ \\
\hline 1 & 4 to 8 & 66.66 & $1 \times 10^{-5}$ to & 180.95 \\
& & & $1 \times 10^{-3}$ & \\
2 & 4.8 to 18.9 & 118.98 & 0.001 to 0.005 & 133.33 \\
3 & 4.7 to 12 & 87.42 & 0.001 to 0.025 & 155.55 \\
4 & 3.9 to 29.4 & 153.15 & 0.01 to 0.02 & 66.66 \\
5 & 2.07 to 2.14 & 3.32 & 0.0013 to 0.25 & 197.93 \\
6 & 7 to 43 & 144.00 & 0.01 to 1 & 196.00 \\
7 & 50 to 105 & 70.96 & 0.0002 to 0.002 & 163.63 \\
8 & 4 to 8 & 66.66 & 0.0001 to 0.002 & 180.90 \\
9 & 5 to 30 & 142.80 & 0.000125 to & 155.50 \\
& & & 0.001 & \\
10 & 10 to 50 & 133.30 & 0.00001 to 0.1 & 199.90 \\
\hline
\end{tabular}

for each structure and can be defined as:

$$
\text { wideness }(\%)=\frac{2 \text { (range maxvalue }- \text { range minvalue })}{\text { range maxvalue }+ \text { range min value }} \times 100 \text {. }
$$

To test the inversion approach in terms of efficiency, values of the relative permittivity and conductivity of 10 structures were selected to reflect relatively large wideness factors. The inversion approach was then applied to the 10 case studies constructed from the available 10 structures. The case studies are summarized in Table 2. To analyze the results of the inversion approach in terms of error, we defined a term for relative percentage error $e_{r}$ as follows:

$$
e_{r}=\operatorname{abs}\left(\frac{\text { True parameter value-Estimated parameter value }}{\text { True parameter value }}\right) \times 100 \text {. }
$$

In order to evaluate the performance of different cooling schedules in terms of error, we calculated the error margin over 100 times for each physical parameter for each cooling schedule as follows:

$$
e_{g}=\frac{\sum_{t} e_{r}}{100}, t=1,2, \ldots . .100
$$

\section{The Two-Dimensional Problem}

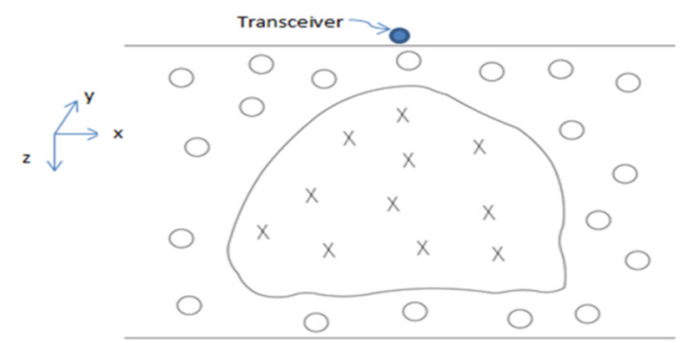

Fig. 5. The 2D model considered in this paper.
Fig. 5 illustrates the $2 \mathrm{D}$ inhomogeneous media used in this paper. The electromagnetic transmitter and receiver are positioned at the same point just above the top of the media with a frequency of one megahertz. For forward problem calculations, circles in Fig. 5 represent an indication of selected points within the slab, while the $X s$ represent an indication of the selected points inside the object. The inversion problem is formulated to estimate four electrical properties of both the slab and the object (i.e., $\left.\varepsilon_{r 1}, \sigma_{1}, \varepsilon_{r 2}, \sigma_{2}\right)$ given the measured scattered electromagnetic fields at $K$ wavenumbers, with $K=11$. The three proposed simulated annealing algorithms were evaluated for the ten $2 \mathrm{D}$ case studies, and an error margin, $e_{g}$, against each case study is shown in Fig. 6 where a comparison is made between each cooling schedule per electrical parameter.

Results in Fig. 6 show that, among the three implemented cooling schedules, the Boltzmann cooling schedule achieves $e_{g} \approx 25 \%$ as the worst value of the tested case studies. On the other hand, the fast and exponential cooling schedules provide $e_{g}>40 \%$, in some tested case studies, although they achieve lower $e_{g}$ values than the Boltzmann cooling schedule in other cases. From the obtained results, it can be concluded that using the Boltzmann cooling schedule within the inversion methodology provides a relatively low error margin for most of the estimated parameters compared with the other cooling schedules. Also, as the $e_{g} \%$ resulting from the Boltzmann cooling schedule are far lower than the wideness factors of the physical parameters, it can be concluded that the inversion methodology achieves better a posteriori knowledge about each physical parameter than the a priori knowledge. The estimated physical parameters can therefore be interpreted correctly after applying the proposed inversion methodology as its estimated values are within its specified range.

\section{The Three-Dimensional Problem}

Fig. 7 illustrates the 3D inhomogeneous media presented in this paper. For forward problem calculations, spheres represent an indication of selected points within the box, while pyramids represent an indication of the selected points inside the object. Here, the transmitter and receiver are located at the same place on top of the media with a frequency of $1 \mathrm{MHz}$. The inversion problem is formulated to estimate four electrical properties for both the object and the box (i.e., $\varepsilon_{r 1}, \sigma_{1}, \varepsilon_{r 2}, \sigma_{2}$ ) given the measured scattered electromagnetic fields at $K$ wavenumbers, with $K=11$. For the presented inversion approach, three simulated annealing cooling schedules were assessed for the 10 proposed 3D case studies. An error margin, $e_{m}$, for each case study is presented and compared in Fig. 8, where a comparison is made between each cooling schedule per electrical parameter. 


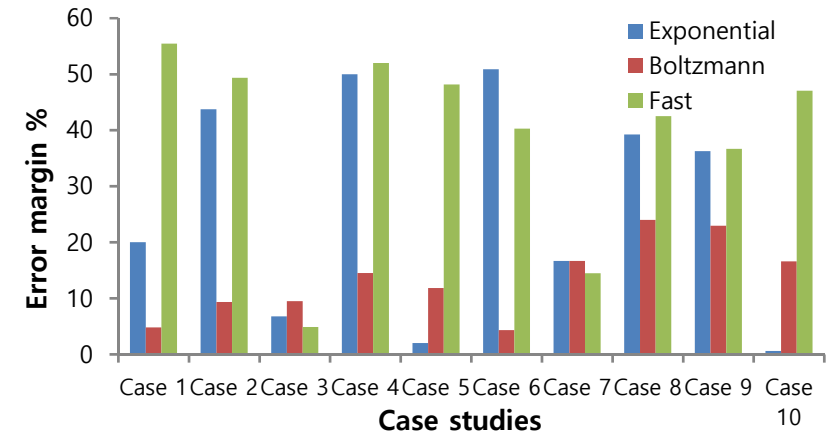

(a)

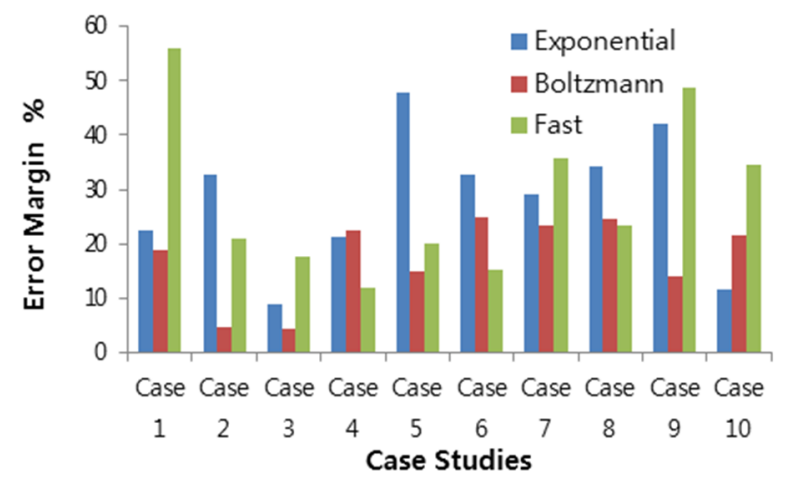

(c)

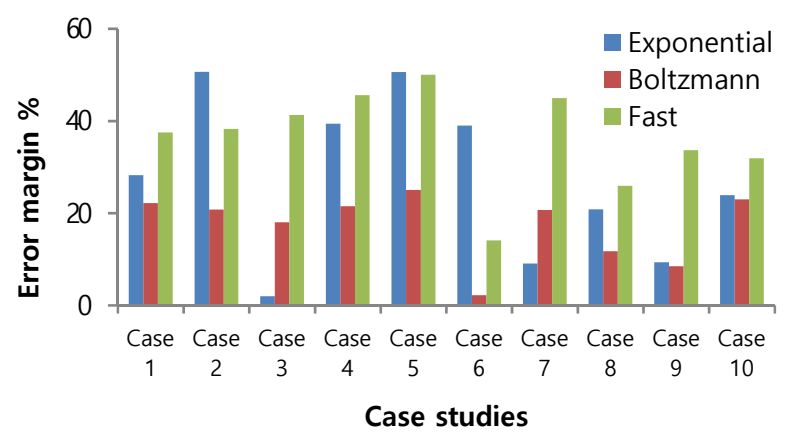

(b)

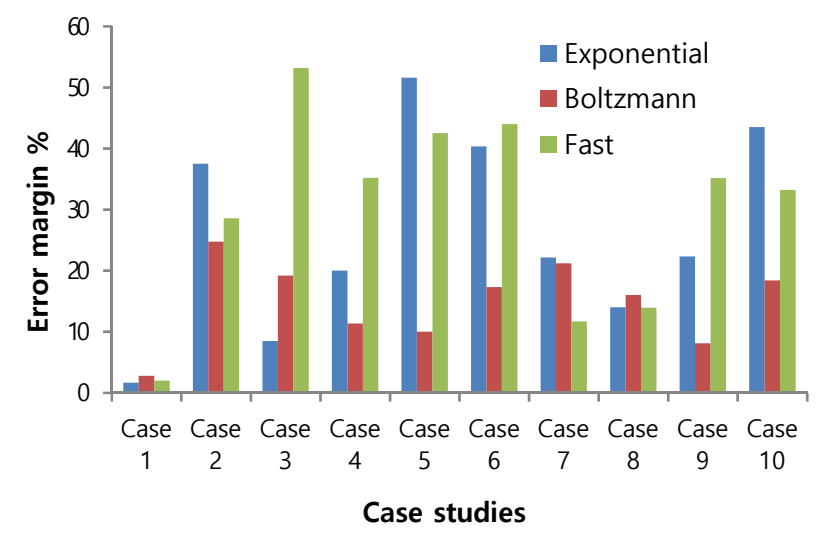

(d)

Fig. 6. Error margins in the 2D problem using exponential, fast, and Boltzmann cooling schedules for the 10 case studies: (a) relative permittivity and (b) conductivity values of the slab; (c) relative permittivity and (b) conductivity values of the object.

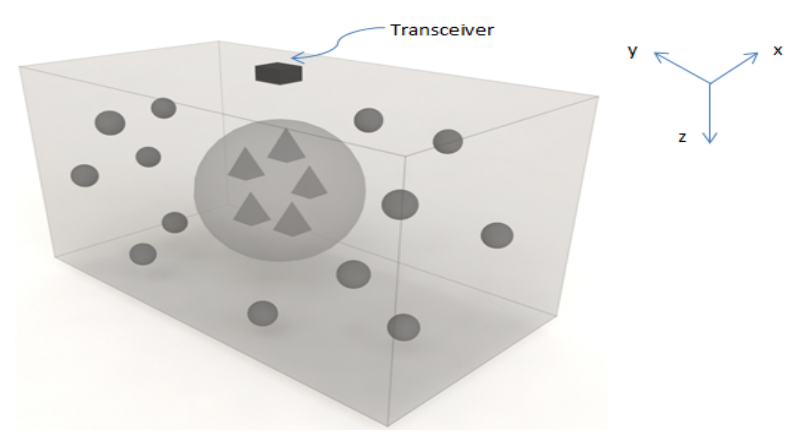

Fig. 7. The 3D model considered in this paper.

Results reveal that $e_{g}$ for the Boltzmann cooling schedule does not exceed $30 \%$ in the worst case among the investigated case studies. The exponential and fast cooling schedules result in a higher $e_{g}$ of $>50 \%$ in the worst-case although they may give a lower $e_{g}$ than the Boltzmann cooling schedule in other cases. It can therefore be concluded that, for the $3 \mathrm{D}$ problem, applying the Boltzmann cooling schedule within the inversion methodology provides better a posteriori knowledge than a priori knowledge. This is due to the relatively low $e_{g}$ of the estimated value compared to the wideness factor of the physical parameters, which in turn results in the correct interpretation of the investigated parameters.

\section{CONCLUSION}

In this paper, an inversion scheme using the simulated annealing technique has been presented to solve both $2 \mathrm{D}$ and $3 \mathrm{D}$ inhomogeneous nonlinear inverse scattering problems. First, a forward model was developed using a meshless formulation to solve Maxwell's equations. The proposed forward model was designed to reduce computational complexity, compared with conventional methods, and therefore to take less time to perform repetitive computations. Second, an inversion approach was proposed to estimate the electric properties of the investigated 2D and 3D scatterers using information about the electromagnetic source and scattered data. Three simulated annealing cooling schedules were evaluated against $2 \mathrm{D}$ and $3 \mathrm{D}$ case studies. The results show that using the Boltzmann cooling schedule gives a worst-case error of $25 \%$ for the $2 \mathrm{D}$ case studies and $30 \%$ for the $3 \mathrm{D}$ case studies. The posterior knowledge of the electric properties of the investigated scatterer is far better than the prior knowledge. These results reveal the efficiency of the presented inversion approach in providing relatively good estimates about the investigated electric properties of the scatterer within their natural range, taking into account the non- 


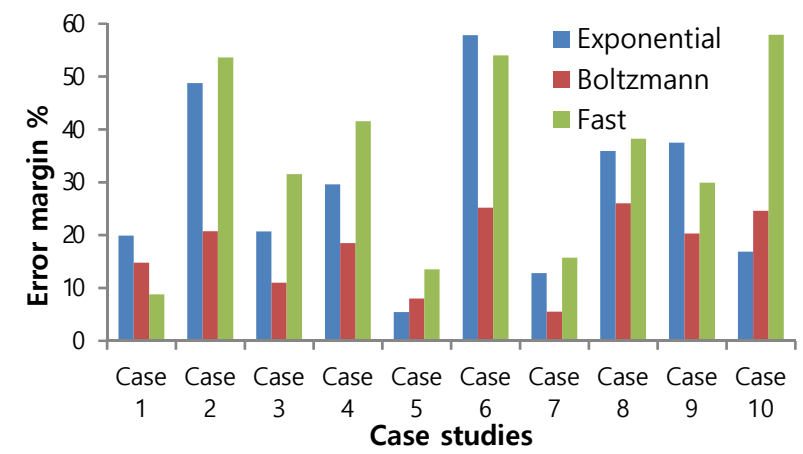

(a)

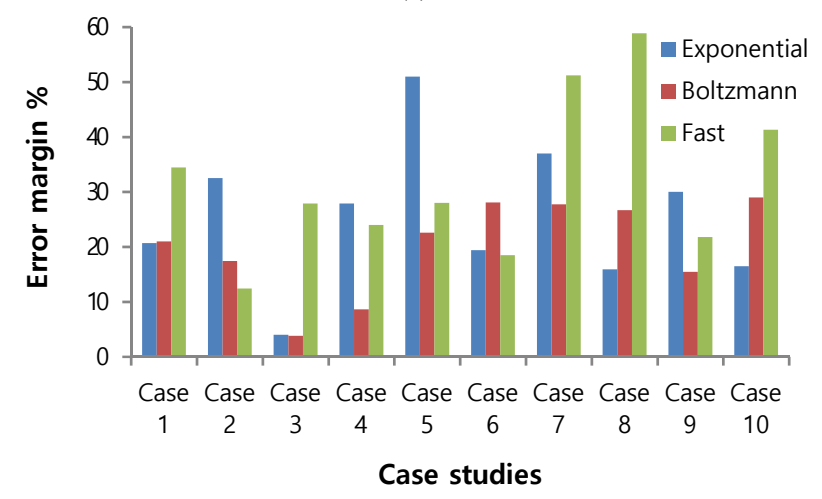

(c)

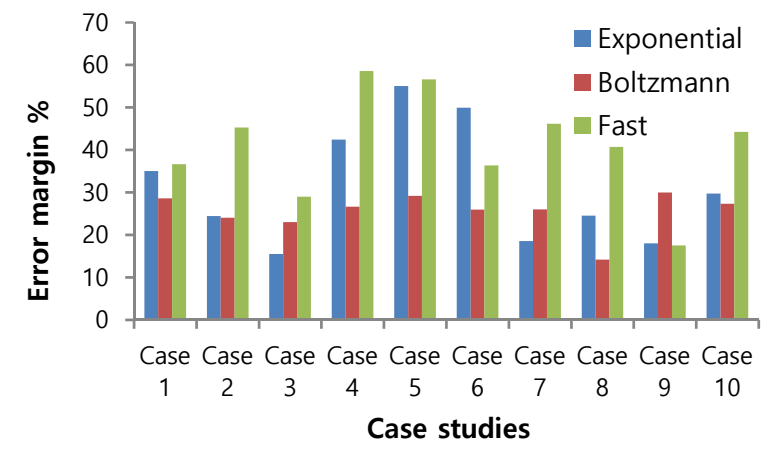

(b)

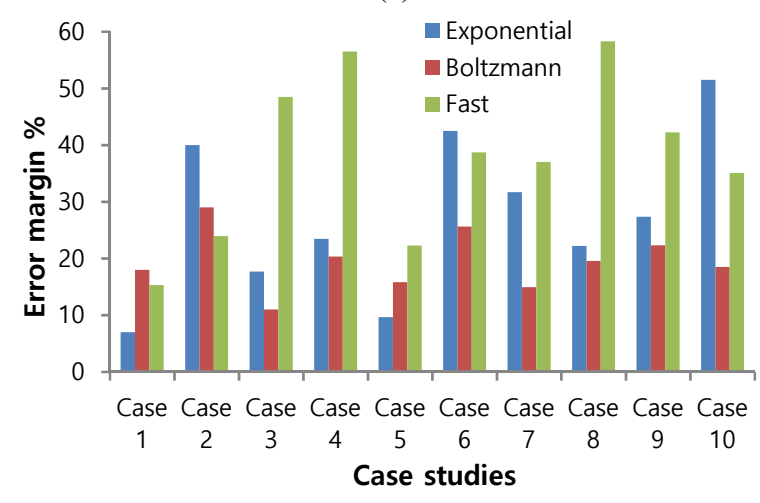

(d)

Fig. 8. Error margins in the 3D problem using exponential, fast, and Boltzmann cooling schedules for the 10 case studies: (a) relative permittivity and (b) conductivity values of the box; (c) relative permittivity and (b) conductivity values of the object.

uniqueness nature of such problems. The proposed approach can be used to solve the inverse scattering problem for inhomogeneous media in different areas of research.

\section{REFERENCES}

[1] H. J. Li and R. Y. Lane, "Utilization of multiple polarization data for aerospace target identification," IEEE Transactions on Antennas and Propagation, vol. 43, no. 12, pp. 1436-1440, 1995.

[2] L. Pellerin, "Applications of electrical and electromagnetic methods for environmental and geotechnical investigations," Surveys in Geophysics, vol. 23, no. 2-3, pp. 101-132, 2002.

[3] J. A. Stratton, Electromagnetic Theory. New York, NY: John Wiley \& Sons, 2007.

[4] N. Pierdicca, P. Castracane, and P. Ciotti, "Inversion of surface scattering models: comparing criteria and algorithms to estimate bare soil parameters," in Proceedings of IEEE International Geoscience and Remote Sensing Symposium, Toronto, Canada, 2002, pp. 1165-1167.

[5] R. C. Aster, B. Borchers, and C. H. Thurber, Parameter Estimation and Inverse Problems. San Diego, CA: Academic Press, 2005.

[6] W. C. Chew and Y. M. Wang, "Reconstruction of twodimensional permittivity distribution using the distorted
Born iterative method," IEEE Transactions on Medical Imaging, vol. 9, no. 2, pp. 218-225, 1990.

[7] A. Dubois, K. Belkebir, I. Catapano, and M. Saillard, "Iterative solution of the electromagnetic inverse scattering problem from the transient scattered field," Radio Science, vol. 44, no. 1, pp. 1-13, 2009.

[8] A. Taflove and S. C. Hagness, Computational Electrodynamics: The Finite-Difference Time-Domain Method, 3rd ed. Boston, MA: Artech House, 2005.

[9] J. Jin, The Finite Element Method in Electromagnetics, 2nd ed. New York, NY: John Wiley \& Sons, 2015.

[10] F. Paris and J. Canas, Boundary Element Method: Fundamentals and Applications. New York, NY: Oxford University Press, 1997.

[11] A. H. Kamel, "Cost-effective schemes for the numerical simulation of electromagnetic waves," Journal of Electromagnetic Waves and Applications, vol. 8, no. 6, pp. 693-710, 1994.

[12] A. Kamel, P. Sguazzero, and M. Kindelan, "Cost-effective staggered schemes for the numerical simulation of wave propagation," International Journal for Numerical Methods in Engineering, vol. 38, no. 5, pp. 755-773, 1995.

[13] M. Birman and M. Solomyak, "On the main singularities of the electric component of the electro-magnetic field in regions with screens," St Petersburg Mathematical Journal, vol. 5, no. 1, pp. 125-140, 1994. 
[14] M. Costabel and M. Dauge, "Singularities of electromagnetic fields in polyhedral domains," Archive for Rational Mechanics and Analysis, vol. 151, no. 3, pp. 221-276, 2000.

[15] F. Yaman, V. G. Yakhno, and R. Potthast, "A survey on inverse problems for applied sciences," Mathematical Problems in Engineering, vol. 2013, article no. 976837, 2013.

[16] S. Caorsi, G. L. Gragnani, and M. Pastorino, "Numerical electromagnetic inverse-scattering solutions for twodimensional infinite dielectric cylinders buried in a lossy half-space," IEEE Transactions on Microwave Theory and Techniques, vol. 41, no. 2, pp. 352-357, 1993.

[17] N. Joachimowicz, C. Pichot, and J. P. Hugonin, "Inverse scattering: an iterative numerical method for electromagnetic imaging," IEEE Transactions on Antennas and Propagation, vol. 39, no. 12, pp. 1742-1753, 1991.

[18] P. Hahner, "Stability of the inverse electromagnetic inhomogeneous medium problem," Inverse Problems, vol. 16, no. 1, pp. 155-174, 2000.

[19] A. Tarantola, Inverse Problem Theory and Methods for Model Parameter Estimation. Philadelphia, PA: SIAM, 2005.

[20] T. M. Monro, D. J. Richardson, N. G. R. Broderick, and P.J. Bennett, "Holey optical fibers: an efficient modal model," Journal of Lightwave Technology, vol. 17, no. 6, pp. 10931102, 1999.

[21] L. B. Felsen and N. Marcuvitz, Radiation and Scattering of Waves. New York, NY: John Wiley \& Sons, 1994.

\section{Mohamed Elkattan}

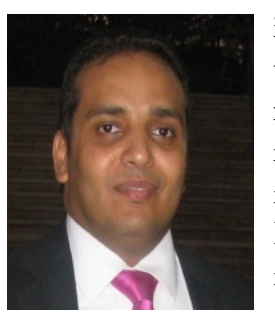

is a lecturer at the Egyptian Nuclear Materials Authority since August 2013. He received his Ph.D. from Ain Shams University, Egypt in 2013. He received his M.Sc. degrees in electronics and communications engineering from Cairo University, Egypt in 2007. His research interests include antennas and wave propagation.
[22] L. Tsang, J. A. Kong, and K. H. Ding, Scattering of Electromagnetic Waves: Theories and Applications. New York, NY: John Wiley \& Sons, 2000.

[23] J. L. Volakis and K. Sertel, Integral Equation Methods for Electromagnetics. Raleigh, NC: SciTech Publishing, 2012.

[24] A. V. Osipov and S. A. Tretyakov, Modern Electromagnetic Scattering Theory with Applications. New York, NY: John Wiley \& Sons, 2017.

[25] D. Colton and R. Kress, "Using fundamental solutions in inverse scattering," Inverse Problems, vol. 22, no. 3, pp. R49R66, 2006.

[26] P. Caro, "On an inverse problem in electromagnetism with local data: stability and uniqueness," Inverse Problems $\varepsilon^{\circ}$ Imaging, vol. 5, no. 2, pp. 297-322, 2011.

[27] J. L. Fernandez Martinez, M. Z. Fernandez Muniz, and M. J. Tompkins, "On the topography of the cost functional in linear and nonlinear inverse problems," Geophysics, vol. 77, no. 1, pp. W1-W15, 2012.

[28] E. G. Talbi, Metaheuristics: From Design to Implementation. New York, NY: John Wiley \& Sons, 2009.

[29] M. Gendreau and J. Y. Potvin, Handbook of Metaheuristics, 2nd ed. New York, NY: Springer, 2010.

[30] M. Elkattan and A. Kamel, "Multiparameter optimization for electromagnetic inversion problem," Advanced Electromagnetics, vol. 6, no. 3, pp. 94-97, 2017. 